\title{
Geographic Information Systems: Usability, Perception, and Preferences of Public Health Professionals
}

\author{
Awatef A. Ben Ramadan, MD, MPH, PhD ${ }^{1,2,3^{*}}$, Jeannette Jackson-Thompson, MSPH, PhD ${ }^{1,2,3}$, \\ Suzanne A. Boren, MHA, PhD,3
}

1. University of Missouri-Columbia (MU): Missouri Cancer Registry and Research Center (MCRARC)

2. University of Missouri-Columbia (MU): School of Medicine, Department of Health Management and Informatics (HMI)

3. University of Missouri-Columbia (MU): MU Informatics Institute (MUII)

*Awatef Ahmed Ben Ramadan, MD, MPH, PhD, aab365@mail.missouri.edu

\begin{abstract}
Background: Analyzing and visualizing health-related databases using Geographic Information Systems (GISs) becomes essential in controlling many public health problems.

Objectives: To explore the perception and preferences of public health professionals (PHPs) about the usability of GISs in public health field

Methods: For this scoping review, the investigators searched Medline Ovid, PubMed, IEEE, Scopus, and GeoBase databases. A total of 105 articles were identified. Nine articles met the inclusion criteria.

Results: Iterative evaluations, training, and involvement of GIS end users are productive in GIS usability. More methodologies are needed to support the validity of GIS usability studies. The exchange of GIS technology impacts public health policy and research positively.

Discussion: PHPs are aware of the use of GISs in the public health field, and the exchange of visualized health data in determining inequalities and inaccessibility issues.

Conclusion: GISs are essential to control public health problems, if the related health datasets are analyzed carefully and if the mapping reports are extensively evaluated and interpreted.
\end{abstract}

Keywords: Geographic Information Systems, GIS, Public Health, Usability 
Correspondence: Awatef Ahmed Ben Ramadan, MD, MPH, PhD, aab365@mail.missouri.edu

DOI: 10.5210/ojphi.v9i2.7437

Copyright (C2017 the author(s)

This is an Open Access article. Authors own copyright of their articles appearing in the Online Journal of Public Health Informatics. Readers may copy articles without permission of the copyright owner(s), as long as the author and OJPHI are acknowledged in the copy and the copy is used for educational, not-for-profit purposes

\section{Introduction}

Public health work requires collaboration and effective communication between team members [1]. Therefore, the Geographic Information System (GIS) tools should be designed to meet the needs and perspectives of the team members. The problem today is not in creating new GISs, but in effective and efficient use of the existing ones [2,3].

Analyzing and visualizing health-related databases, using sophisticated statistical software, is essential in helping control many public health problems in any community. This data should be handled carefully, analyzed adequately to get reliable results, and not mislead the target audiences [4].

Most of the potential users of the health-related spatial data find difficulties in interpreting statistical and mapping information of most health-related spatial reports [5-7]. The major issues are lack of experience and training to use this technology, lack of acceptance to use GIS tools, and complicated design of most existing GIS technologies [8]. Providing the potential users of GIS software with clear explanations on the statistical methodology and results and analogies of the combined diagrams and maps will enhance the users' understanding and motivate them to use this technology [9]. Mapped public health data can create knowledge, produce evidence, and generate policies [10]. Every mapping report should carry a specific purpose and carry a clear message to the audience [11]. Targeting the public health professionals (PHPs) and policy makers, the mapping reports should include citations of the used databases' sources and the methodology of the results. In order to make it user friendly, the usability of the GIS tools and reports should be iteratively pilot tested by potential users before and after tools' release [4].

Current literature proves the collaboration between the professionals of the same public health interest by linking health information from different sources and designing portals and applications [12]. This will help guide PHPs and policy makers to develop cost-effective public health interventions [12]. Over the last 20 years, spatial health data are transformed from being static to being interactive and dynamic [13].

GIS tools could help communication between experts in different fields. The GIS developers and users should consider technical, social, and cultural issues during development, evaluations, and updates of the GISs tools to enhance the experts' connection [14]. The investigators of the current 
scoping review could not find previous literature reviews adopting the same aim, including all of this review's inclusion criteria (see methods section), covering exactly the time limits of this review, and/or using the same searching strategies and similar keyword terms used in the current review.

The current scoping review's aim is to explore the perceptions and preferences of public health practitioners and policy makers about the use of GISs in public health practice, and to search the literature about the usability and utility of GISs in public health fields.

\section{Methods}

\section{Study Design and Search Strategy}

The study design was a scoping review research. The investigators initially searched for eligible journal articles in the following databases: Medline Ovid and PubMed databases. The following terms were searched using Medline Ovid: 1) Geographic Information System (GIS) OR GIS OR mapping software, AND 2) public health OR public health practitioners, AND 3) usability OR functionality OR utility OR perception OR preferences. The search resulted in two articles. The PubMed was searched with the same strategy and did not produce any results. The investigators tried to search the same terms differently using PubMed database. By using the strategy: Geographic Information Systems AND public health AND perception AND GIS, our search results produced 35 articles, including just one article which is strongly related to the current study aim. The article's title was "Interactive map communication: pilot study of the visual perceptions and preferences of public health practitioners" [15]. The investigators searched the article's references and the article's similar articles which were listed on the right side of the article's abstract PubMed page. From both the Medline Ovid and the unique PubMed strategy, we identified a total of 103 articles.

The investigators searched the Institute of Electrical and Electronics Engineers (IEEE) ${ }^{\circledR}$ Xplore database using the same terms and the search produced two articles. The Scopus and the GeoBase databases also were searched using the same terms without producing any related articles.

The collected 105 articles were screened by reading their abstracts and 48 articles were excluded because their objectives did not meet the review's aim. The investigators assessed the eligibility of the selected 57 strongly related articles by reading the articles' full text. At the end, nine articles met the review's inclusion criteria (Figure 1).

\section{Inclusion Criteria}

Eligible articles for this review were written in English, published in the years from 2000 to 2016, and included usability interviews or usability testing of public health professionals (PHPs) and/or decision- or policy-makers. In the usability testing literature, the studies test the usability of: GISs, mapping atlases, mapping applications, or spatial or spatial-temporal websites and/or portals. These mentioned tools should display spatial or spatial-temporal public health data. In the usability studies, the inclusion criteria for the users were public health practitioners or professionals, 
epidemiologists, public health program directors, spatial reports developers and analyzers, and public health policy makers.
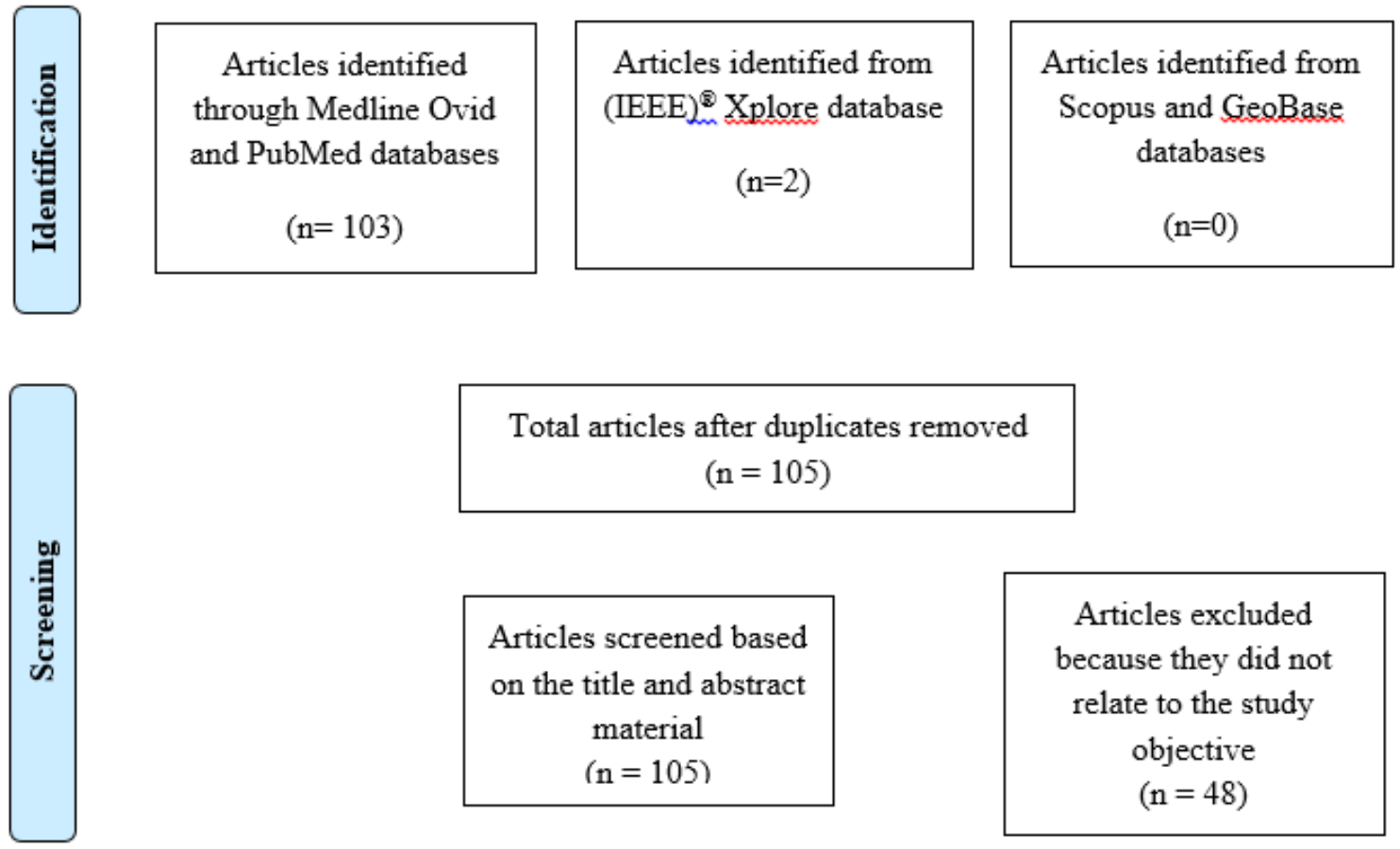

Total articles after duplicates removed

$$
(n=105)
$$

Articles screened based
on the title and abstract
material
$(\mathrm{n}=105)$

Articles excluded because they did not relate to the study objective $(n=48)$
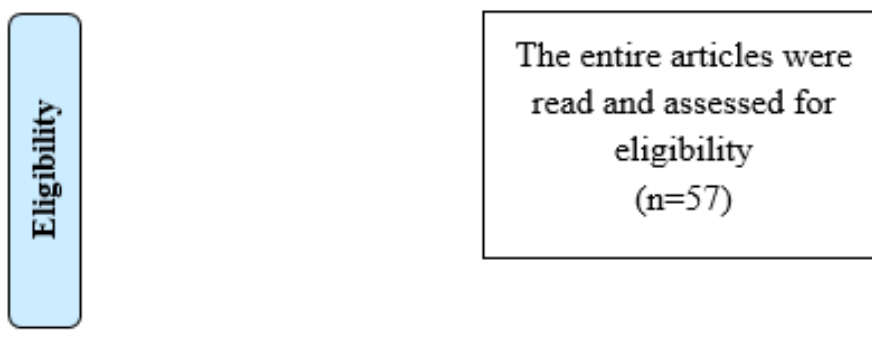

Excluded after reading complete article $(\mathrm{n}=48)$

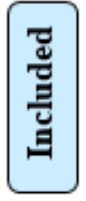

$$
\begin{aligned}
& \text { Articles included in the } \\
& \text { scoping review } \\
& (\mathrm{n}=9)
\end{aligned}
$$

Figure (1): Search Strategy Flow Chart

According to the study design and the methodology, we divided the eligible articles into:

1. Articles based on usability testing of GIS tools, applications, and/or websites display of spatial-temporal data: Four articles met the study inclusion criteria. 
2. Articles based on interviewing PHPs and policy-makers to find out their GISs' perspectives and preferences in public health field: Five articles met the study inclusion criteria.

\section{Results}

There is variety among the nine eligible articles presented in Table 1 and Table 2 . The participants are different (demographics, experience, work type, and degree of education). Different GIS software is tested and different research measures are used. See Table 1 and Table 2.

\section{Usability Testing Studies:}

There are four articles in this category. The important information about the studies was extracted and presented in Table1.

The first study was conducted by a geography scholar. The study's objective was to explore how epidemiologists take advantage of the geo-visualized technology, and how they expect this information to help them in practice. The study design was usability testing of the Exploratory Spatial-Temporal Analysis Toolkit (ESTAT) which visualizes multivariate health data to support cancer epidemiology. The study was user-centered and the researchers conducted iterative evaluation processes to refine ESTAT.

The study design was multi-staged. In the first stage, graduate students used card sorting and verbal protocol analysis. After a year, the study investigators shifted to the actual end-users after they had problems with the tool's interface. In the second stage, the researchers conducted verbal protocol analysis on 12 epidemiologists followed by focus group activities to discuss the testing usability sessions. Verbal protocol is defined as: "A psychological research method that elicits verbal reports from research participants" [16]. In the third stage, a case study in collaboration with an academic epidemiologist was conducted to analyze ESTAT. The analysis was a positive addition to the tool's design. In the fourth stage, five experts in data analysis tested the refined tool using verbal protocol analysis followed by focus groups. A scatter plot was the first analytical measure the epidemiologists used followed by bivariate map tools. The complicated and multivariate tools of the ESTAT were not used commonly with the users. The most interesting finding of this stage was that the users did not face a lot of interaction problems, and this indicated improvement in development and refinement of the tool [17]. 


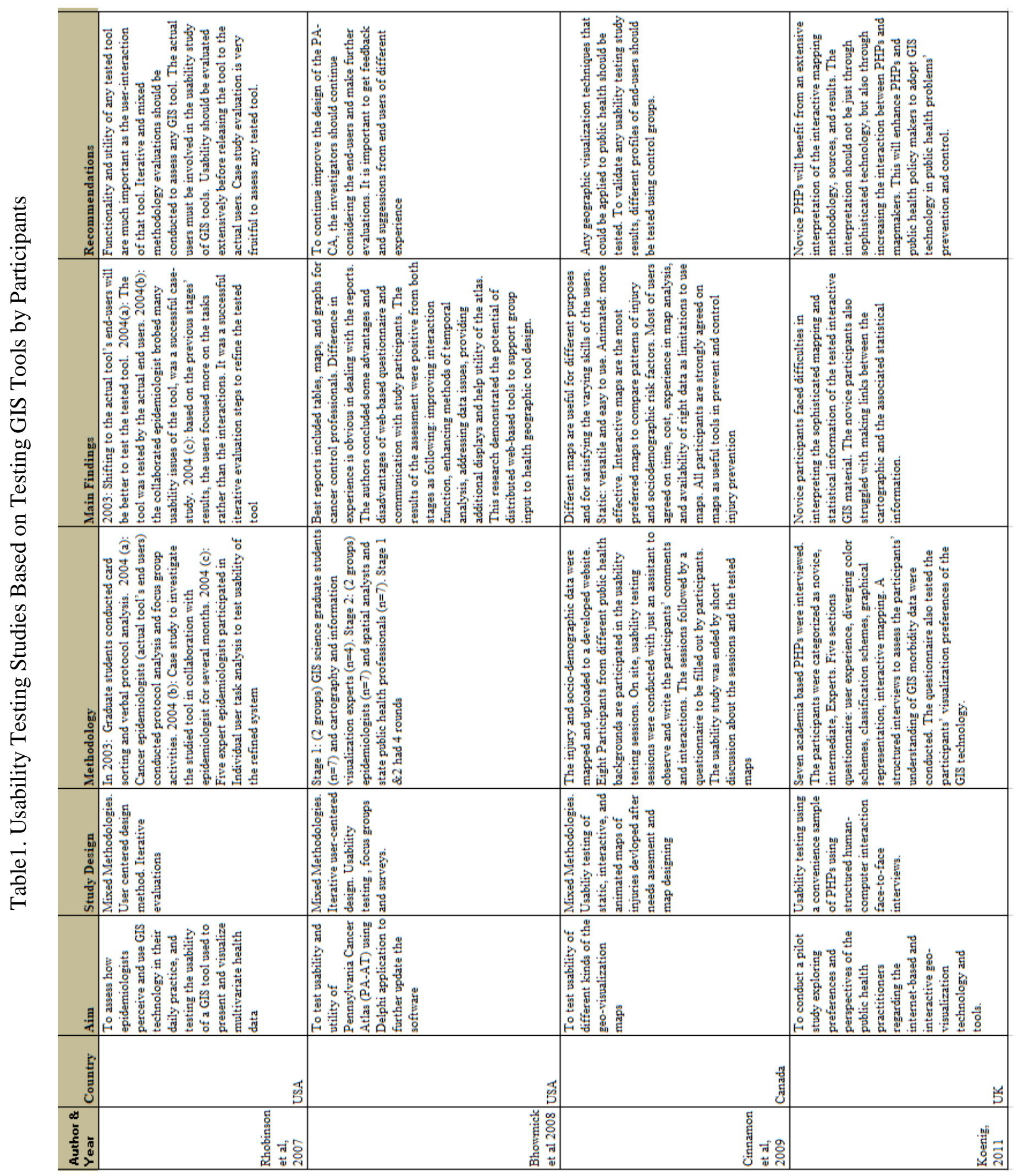


The second study was conducted by scholars from four different specialties and expertise: public health, geography, clinical medicine, and cancer research. The study objective was to test the usability and utility of the Pennsylvania Cancer Atlas (PA-CA) to refine the software. The study design was multi-staged user-centered evaluations of PA-CA usability using web-based application (Delphi application). In the first stage, the investigators tested the PA-CA using two groups of users. The first group included seven GIS science graduate students, and the second group formed from four cartography and information visualization experts. The second stage included two groups, seven epidemiologists in the first group, and seven spatial analysts and Pennsylvania state public health professionals in the second group. Every stage of evaluation had four rounds of testing sessions. The professional participants pointed out that the best spatial reports included tables, maps, and charts. The responses and the using of the tested spatial reports were varied by the difference in expertise. Most of the participants stressed the importance of integrating tutorials and help information for the PA-CA end-users. The results of the evaluation processes were totally positive. The testers came up with important recommendations on the PACA software: improving user-software interface and motivating new methods of temporal analysis. The other main finding of the study was the ability to distribute web-based tools to access different kinds of experts and recruit them to test the design of GIS tools [18].

The third study was conducted by researchers of different scientific backgrounds: geography, medical school, and public health specialties. The study design was multi-staged. First stage: There was a user needs assessment, through meetings with public health stakeholders who described need for injury-related GIS tools and reports. Second and third stages: These combined stages were named as the map development stage. Three map types were created by the researchers; the maps were: static, animated, and interactive maps. The created maps displayed the injury data and its socio-demographic determinants. These maps were uploaded to a developed website. Fourth stage: The uploaded maps were tested using a sample of public health officials and injury prevention stakeholders. The usability testing sessions were on-site with an observer to write down the users' comments and their map-interface. The sessions were followed by a self-filled-out questionnaire and short discussion per participant. All the participants revealed that all map types are useful for different purposes. Most of them pointed out that the animated maps are more effective than the static maps, and the best maps to effectively compare the injury data to its socio-economic determinants were the interactive maps. Most of the users agreed on the effect of the resources in terms of time, money, expertise on the map development industry, and the availability of right and appropriate data to build successful maps [19].

The fourth study was carried out by three scholars from different specialties and educational institutions: Public health science, environmental and engineering science, and geography science. The study aims were to conduct a pilot study on a sample of PHPs to explore their comprehensive and visualization preferences of the interactive online-based mapping reports and to evaluate the effectiveness of the interactive mapping reports' formats and measure the actual end-users' interactions with the tested GIS tools.

The study design was built on a five-section interview questionnaire. The test was on-site, faceto-face, and a GIS-based interview. The interviews were accompanied with direct observation and a think-aloud protocol. The participants were asked to examine the tested visualization material, answer the questions, and write down their preferences, perceptions, and expectations on the tested 
material. Seven academic PHPs were assigned, according to their expertise of using disease visualization maps, based on their answers on a specific question to novice, intermediate, and expert categories. The interviews included five sections in a well-structured questionnaire: User experience, diverging color schemes, data classification schemes, graphical representation of morbidity data, and interactive mapping usability tasks. The novice participants had problems in exploration of the data classifications, in understanding the supplementary sophisticated statistical graphics, and in linking the interactive tables with the maps of the tested reports. There were differences in the perception of the interactive mapping reports among the participants according to their previous geographic experience [15].

\section{Usability Studies Based on Just Interviewing the Participants:}

There are five articles under this category. The articles' methodologies were based on interviewing PHPs, cartographic scientists, map developers, and/ or public health policy makers. See Table (2).

The first study was conducted as a collaboration of three geography scientists from three different universities. The aim of the study was to analyze the organizational issues which are important to successfully implement GISs within the National Health Service (NHS) in the UK and compare the results to previous studies that were conducted to analyze the same aim. The study design was mixed-methods, starting with a national questionnaire followed by semi-structured interviews. The national questionnaire was formed on the current use of GIS software, future plans for GIS use, policy-related uses of GIS, barriers to using GIS, and enumerates human, environmental, and organizational barriers to implement GIS. The questionnaire was answered by health services professionals. An in-depth interview was conducted on 20 selected NHS personnel. The interviews included the potential issues to establish GIS software: individual issues, policy issues, data issues, organizational issues, and various resources issues. The national survey revealed an increase of GIS use, map production and GIS use in analysis, modeling, and data integration are important. The examples of GIS uses were in: inequalities, accessibility determination, and environmental sciences. Less than $50 \%$ of the interviewees stated they did not fully operate their GISs. Informational technology administration and maintaining systems are influential for GIS implementation. Both the survey and the interviewees stated a list of the obstacles to GIS implementation: lack of digital data, difficult analytical tasks of GIS, lack of staff resources to operate GIS, lack of GIS skills, lack of maintenance systems, lack of wide organizational planning, lack of authority's awareness, insufficient training of the GIS users, lack of central plan and support from the department of health to its organizations, and lack of awareness among clinicians and administrators of GIS importance. The respondents were asked about the barriers and issues which restricted the geographic information exchange. Responses were varied: Licensing arrangement issues between the organizations, presence of GIS data in specific formats, lack of interest of GIS exchange in other organizations, and hardware and software incompatibilities among different organizations [20]. 
Table 2. Usability Studies Based on Interviewing the Participants

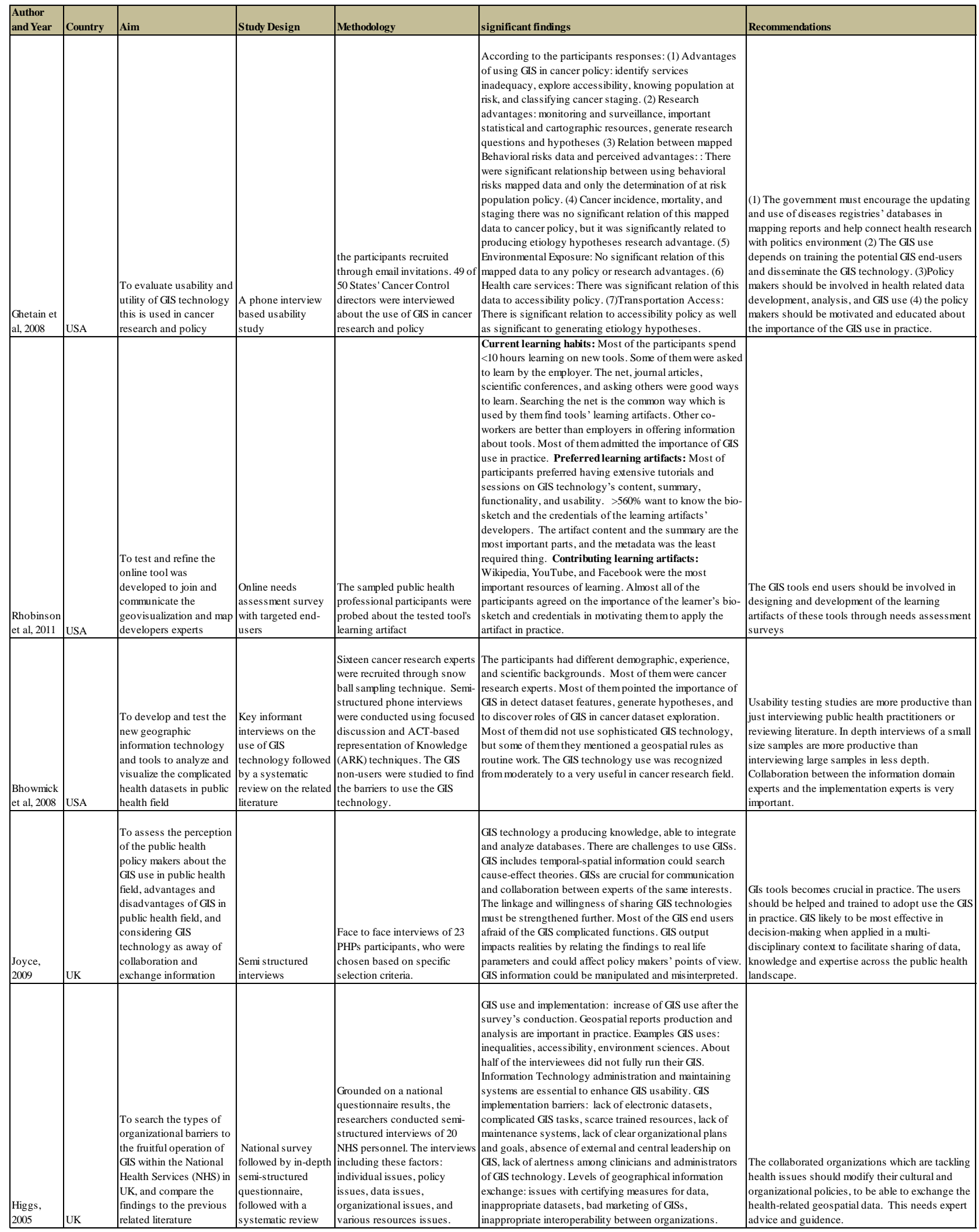


The second study was conducted by three communication and art scientists and a public health scientist. The aim of the study was to evaluate the use and the utility of GIS tools in mapping cancer-related data and their effect on cancer control policies and practice, and to measure the participants' perception on using such tools in the Comprehensive Cancer Control (CCC) program. The researchers recruited participants through email. Forty-nine U.S. CCC program directors out of 50 states were interviewed by phone. The interview questions were to explore the relationship of GIS reports to public health policy and research. The identified advantages of using GIS on cancer policy were: Identify service gap, identify access issues, identify cancer staging, followed by identify risk population. The identified advantages of using GIS on cancer research were: Multivariate modeling tool, monitoring and surveillance, followed by generate etiologic hypotheses. There was no significant relationship discovered between using behavioral risks mapped reports and research, while there was a significant relationship between behavioral risks mapping data and the policy of identifying the at risk population. The study did not discover any significant relationship of cancer burden mapped reports and cancer control policy, but there was a significant relationship of this kind of mapped reports and the generating etiology hypotheses research advantage. About $51 \%$ of the interviewed directors stated that the demographics are an important content of any mapped reports, but the study researchers could not find any significant relation of the demographics to any of the policy and research advantages. There was significant relation of mapped reports of transportation access and the accessibility policy, and the etiology hypotheses research advantages. There were no significant relations between policy and research, and all of these kinds of cancer mapped contents: Environmental exposure, multi-layer content maps, and healthcare services [21].

The third study was conducted by scientists from geography, environmental science, and public health fields. The aim of the study was to develop and evaluate the tools and methods that might be used by PHPs in order to extract knowledge and evidence from health-related databases. The methodology of the study was semi-structured phone interviews with 16 participants who were recruited using snowball sampling. The investigators searched the literature of using GIS in cancer research to support the interviews' results. Most of the participants were faculty or senior administrators of different demographic and scientific backgrounds. They varied in experience. Most of the participants were involved in the cancer research domain. Most of the participants pointed out that the typical goals of data exploration were to detect dataset aspects, to develop hypotheses for further cancer research, and to discover roles of geospatial methods in the exploration process. Most of them did not use complex spatial analysis, but 30\% of them reported geo-coding, map creation, and GIS data analysis as regular research activities. GIS analyses were considered from moderate to very useful tools in cancer research, specifically in incidence and mortality cancer data. They pointed to the importance of GIS in comparing spatial data of different cancer types, disease clustering, correlation with related spatial indicators, and combining geospatial data from different domains. The participants pointed to the following limitations in GIS use: Difficulties in geo-coding and data aggregation, lack of support for merging data from different data sources and/or constructed with different GIS tools, complexity of GIS tools use and functionalities [22].

The aim of the study described in the fourth article was to study public health professionals' perceiving of the GIS in practice and research and understand the impact of GIS on data sharing and communication. The methodology was face-to-face semi-structured interviews with 23 
participants who were policy decision makers. The participants were recruited purposefully. The article findings are: GISs are converting raw data to useful data and knowledge. GIS has the ability to integrate and analyze datasets. GISs are important in public health practice and decision making but include many implementation and usability challenges. GIS could be used to explore causeeffect relationship by including time and space and have epidemiological power. GISs are crucial for collaboration between experts of the same interests but there are challenges to that. The linkage and willingness of sharing GIS technologies must be strengthened further. Most of the low experiences in GIS use of public health practitioners are fear of the sophisticated functions of the GIS tools. GIS output impacts realities and could affect policy makers' decisions. Some participants pointed out that GIS tools are not neutral and map makers might manipulate datasets using GIS power. Metadata and detailed text are very important to interpret the GIS data. Data quality is very important as well as strict standards during constructing GIS data. GIS tools are considered user-friendly and easier to relate data tools. Time and resource constraints, training skills, and intra-organization environments enhance feelings of insecurity and concern among potential GIS end-users [23].

The last article's aim was to conduct a needs-assessment survey on the potential end users of the Geo-visual Explication (G-EX) portal, which is an online tool designed to connect researchers in geo-visualization to the end users, to refine the G-XL Learn module. The researchers developed a web-based survey using their previous in-depth usability studies. The participants were recruited by sending emails. There were 21 participants from different backgrounds: Epidemiologists, health policy specialists, geographers, and research scientists. The results were as follows: Most of the participants spend less than ten hours per week learning new tools and $20 \%$ of them were required by their employers to keep learning these tools. The ways of learning about new tools were: the internet, journal articles, conference sessions, and asking colleagues. The least likely ways participants learned about these tools were advertisements and employer contribution. The participants' preferred learning artifacts were comprehensive tutorials followed by hands-on training. Most of the participants wanted the artifacts to include expected training duration and summary of learning objects, and they preferred to start using the software before starting the training. $63 \%$ of the participants liked to know the biography of the trainers. Most of the participants spotted that the artifact's content, summary, and the instructions are the most important parts of any learning module. The other contributing artifacts for the participants were: Wikipedia, YouTube, and social media, respectively. Fifty two of the participants were interested in development of training material to share with others on the G-EX website [24].

\section{Discussion}

The findings of the reviewed articles are discussed separately as they were classified in the methods and the results sections.

\section{Usability Testing Studies}

It is important to include a representative sample of the actual users in any usability testing of GIS tools. A five-participant study can demonstrate most of the usability problems of the tested material [25]. This enables the investigators to measure the actual user-tool interface and helps them successfully design, implement, and refine these tested tools. All the studied usability testing 
articles used the actual users to test the GIS systems. The review also revealed that the usability testing research should extend to explore the content, functionality, and utility of the GIS tools. The review stated that any GIS tool should be iteratively evaluated using different methodologies. The review discovered that case studies by collaboration with experts were very valuable in development and refinement of GIS tools. The review concluded that visualizing the health-related data in an interactive way, including tables, maps and graphs, is considered the best way to present such information. The review revealed the importance of the development of online applications to access more potential users and help them participate in testing the GIS tools. The review stated that building successful mapping reports depends on the availability of monetary support, right data, and expertise in map construction. The review pointed out that the level of experience in using data visualization is critical for being willing to use GIS software and interpretation and linking of the mapping reports' information.

\section{Usability Studies Based on Just Interviewing the Participants}

The review revealed that even to assess the PHPs' preferences and perspectives the researchers do not have to rely on just interviewing the participants, but they need to search for more methodology to support the validity of their results. In some of our reviewed articles, researchers supplemented their interviews with the results of well-respected national questionnaires and some did systematic reviews to support the study evidence and generalizability.

Health organizations should assess and overcome the organizational, cultural, technical, and expertise barriers to implement and use GIS software to visualize their data. One of our reviewed articles recommended the adoption of policies that support visualization of health-related data on the state level and valued the importance of state encouragement of utilization and presentation of disease registries on geographic bases to connect health research to the political environment. All the reviewed articles pointed to the importance of dissemination of successful GIS technology, training the potential users adequately and giving up-to-date information technology administration support and maintenance. Most of the reviewed articles recommended involvement of policy makers in using GIS tools and in analysis of the GIS tools results. The review recommended collaboration between GIS software developers and implementers and potential end-users to develop new, and test refined, versions of public health GIS tools.

The review articles in this section revealed that needs assessment is crucial to know the perspectives of the GIS potential users and to develop web learning portals and modules. The review suggested that the learning artifacts of GISs tools could be presented in different formats based on users' preferences. The review recommended that employers offer extensive training for their GIS potential users before and after implementation of the GIS tools.

\section{List of Recommendations Learned from the Review}

The review's investigators constructed a list of recommendations they learned from conducting the review. This list could help other researchers conducting similar reviews. It also might help public health practitioners to decide on the type of information and the way they should visualize the health-related data to satisfy potential users. The list might help the GIS technology designers, builders, and vendors to develop a user-friendly technology by tailoring the developed technology 
according to their users' preferences and insights. Figure (2) shows the recommendation list we learned from the current review.

\section{Recommendations from Review Methodology:}

- For a relatively narrow and an innovative field of study, as we have in this review, the investigators should search a wide array of interdisciplinary databases to come up with the maximum collection of literature records to help conduct a detailed scoping review of the related published articles.

- For scarce search results, the investigators also should try different ways of searching the targeted databases in order to allocate as much as they could of the related articles.

2. Recommendations from the Studied Articles:

- GIS might be operational in decision-making through facilitating the exchange of healthrelated data and information across the different public health disciplines.

- The health facilities must modify and update their cultural and structural rules, to be able to interchange the health-related interactive geographical reports and maps.

- The government should boost the updating and usage of diseases registry databases in producing diseases atlases and facilitate connecting research with policies setting.

- Utility of any GIS technology is as critical as the usability of the tool.

- Extensive usability and utility assessments are essential to evaluate the tools before and after they are released to the actual users.

- A representative sample of actual users should be included in the usability study of any tested GIS tools.

- It is essential to collect novice and the expert users' feedback and suggestions about the GIS tools they use.

- To confirm any usability testing study outcomes, we should use a diverse sample of end-users to match with a control group.

- Beginner users must be trained extensively and should be provided with a broad interpretation of every new technology tool.

- The interaction between PHPs and mapmakers should be strengthened to enhance PHPs and public health policy makers to adopt GIS technology in the public health field.

- Policy makers should be involved in health related data development, analysis, and GIS use.

- Usability testing methodology is more fruitful than questioning public health practitioners and conducting reviews.

- In-depth structural and semi-structural interviews of a small size samples of the GIS technology users are more beneficial than questioning large samples in less depth.

Figure (2). List of Recommendations Learned from the Review 


\section{Conclusion}

In general, the review revealed that PHPs are aware of the importance of using GIS software on public health policy and research. In most of the studies, participants pointed to the advantages of using GISs on public health practice to determine inequalities and accessibility. They also stated the importance of supplementary roles of other contextual indicators on different public health problems when these indicators are visualized with the health-related data.

Most of the studies revealed the participants were aware of the collaboration and the exchange of the GISs technology and data between experts in the public health field, and the importance of including the end users in the basic stages of design and development of GIS tools. The participants were also aware of the importance of extensive evaluations for GIS tools before and after releasing them and the essential need for training the potential users of these GIS tools.

\section{Review Strengths, Limitations and Future Directions}

Due to the innovative review's purpose, the review's authors targeted specific databases which could produce a maximum number of scientific-based articles to match the current review's aim. This is a considerable strength for this study. To get a detailed and updated scope of the searched literature, the investigators used strict inclusion criteria and used the literature published in a limited period of time. The investigators wanted to produce an in-depth analysis of an innovative field of study and help future researchers comprehend GIS use in public health and the ideal way of conducting usability studies to assess this technology. Future usability testing studies must include more potential end users who must be recruited randomly and tested using more sophisticated methodology, as well as quantitative and qualitative measures.

\section{References}

1. Sicotte C, D’Amour D, Mordaunt MP. 2002. Interdisciplinary collaboration within Quebec community health care centres. Soc Sci Med. 55(6), 991-1003. PubMed https://doi.org/10.1016/S0277-9536(01)00232-5

2. Brooks Jr FP. The Mythical Man-Month: Essays on Software Engineering, Anniversary Edition, 2/E. Pearson Education India; 1995 Sep 1.

3. Young RR. Effective requirements practices. Addison-Wesley Longman Publishing Co., Inc.; 2001 Mar 1.

4. Bell BS, Hoskins RE, Pickle LW, Wartenberg D. 2006. Current practices in spatial analysis of cancer data: mapping health statistics to inform policymakers and the public. Int J Health Geogr. 5(1), 49. PubMed https://doi.org/10.1186/1476-072X-5-49

5. Lipkus IM, Samsa G, Rimer BK. 2001. General performance on a numeracy scale among highly educated samples. Med Decis Making. 21(1), 37-44. PubMed https://doi.org/10.1177/0272989X0102100105 
6. Sheridan SL, Pignone M. 2001. Numeracy and the medical student's ability to interpret data. Effective clinical practice. ECP. 5(1), 35-40. PubMed

7. Woloshin S, Schwartz LM, Moncur M, Gabriel S, Tosteson AN. 2001. Assessing values for health: numeracy matters. Med Decis Making. 21(5), 382-90. PubMed https://doi.org/10.1177/0272989X0102100505

8. Rogers EM. Diffusion of innovations. Simon and Schuster; 2010 Jul 6.

9. Woloshin S, Schwartz LM, Zellner A. 2003. Making sense of risk information on the web. BMJ. 327(7417), 695-96. PubMed https://doi.org/10.1136/bmj.327.7417.695

10. MacEachren AM. How maps work: representation, visualization, and design. Guilford Press; 2004.

11. Frye C. 2001. Making maps that communicate. ArcUser. 4, 38-43.

12. Richards TB, Croner CM, Rushton G, Brown CK, Fowler L. 1999. Information technology: Geographic information systems and public health: Mapping the future. Public Health Rep. 114(4), 359. $\underline{\text { PubMed https://doi.org/10.1093/phr/114.4.359 }}$

13. Pickle LW. Atlas of united states mortality. National Center for Health Statistics, Centers for Disease Control and Prevention, US Dept. of Health and Human Services; 1996.

14. Paolino L, Sebillo M, Cringoli G. 2005. Geographical information systems and on-line GIServices for health data sharing and management. Parassitologia. 47(1), 171. PubMed

15. Koenig A, Samarasundera E, Cheng T. 2011. Interactive map communication: Pilot study of the visual perceptions and preferences of public health practitioners. Public Health. 125(8), 554-60. $\underline{\text { PubMed https://doi.org/10.1016/j.puhe.2011.02.011 }}$

16. Crutcher RJ. 1994. Telling what we know: The use of verbal report methodologies in psychological research. Psychological Science-Cambridge. 5, 241. https://doi.org/10.1111/j.14679280.1994.tb00619.x

17. Robinson AC. 2007. A design framework for exploratory revisualization in epidemiology. Inf Vis. 6(3), 197-214. PubMed https://doi.org/10.1057/palgrave.ivs.9500155

18. Bhowmick T, Robinson AC, Gruver A, MacEachren AM, Lengerich EJ. 2008. Distributed usability evaluation of the Pennsylvania Cancer Atlas. Int J Health Geogr. 7(1), 36. PubMed https://doi.org/10.1186/1476-072X-7-36

19. Cinnamon J, Rinner C, Cusimano MD, Marshall S, Bekele T, et al. 2009. Evaluating web-based static, animated and interactive maps for injury prevention. Geospat Health. 4(1), 3-16. PubMed https://doi.org/10.4081/gh.2009.206 
20. Higgs G, Smith DP, Gould MI. 2005. Findings from a survey on GIS use in the UK National Health Service: organisational challenges and opportunities. Health Policy. 72(1), 105-17. PubMed https://doi.org/10.1016/j.healthpol.2004.06.011

21. Ghetian CB, Parrott R, Volkman JE, Lengerich EJ. 2008. Cancer registry policies in the United States and geographic information systems applications in comprehensive cancer control. Health Policy. 87(2), 185-93. PubMed https://doi.org/10.1016/j.healthpol.2007.12.007

22. Bhowmick T, Griffin AL, MacEachren AM, Kluhsman BC, Lengerich EJ. 2008. Informing geospatial toolset design: understanding the process of cancer data exploration and analysis. Health Place. 14(3), 576-607. PubMed https://doi.org/10.1016/j.healthplace.2007.10.009

23. Joyce K. 2009. "To me it's just another tool to help understand the evidence": Public health decisionmakers' perceptions of the value of geographical information systems (GIS). Health Place. 15(3), 83140. PubMed https://doi.org/10.1016/j.healthplace.2009.01.004

24. Robinson AC, MacEachren AM, Roth RE. 2011. Designing a web-based learning portal for geographic visualization and analysis in public health. Health Informatics J. 17(3), 191-208. PubMed https://doi.org/10.1177/1460458211409718

25. Albert W, Tullis T. Measuring the user experience: collecting, analyzing, and presenting usability metrics. Newnes; 2013 May 23. 